\title{
Using Telehealth to Deliver Primary Care to Adolescents During and After the COVID-19 Pandemic: National Survey Study of US Primary Care Professionals
}

Melissa B Gilkey ${ }^{1}, \mathrm{PhD}$; Wei Yi Kong ${ }^{1}$, MA; Qian Huang ${ }^{1}, \mathrm{PhD}$; Brigid K Grabert ${ }^{1}$, PhD, JD; Peyton Thompson ${ }^{2}$, MScR, MD; Noel T Brewer ${ }^{1}$, PhD

\footnotetext{
${ }^{1}$ Department of Health Behavior, University of North Carolina at Chapel Hill, Chapel Hill, NC, United States

${ }^{2}$ Department of Pediatrics, University of North Carolina at Chapel Hill, Chapel Hill, NC, United States
}

\section{Corresponding Author:}

Melissa B Gilkey, PhD

Department of Health Behavior

University of North Carolina at Chapel Hill

CB 7440

Chapel Hill, NC, 27599

United States

Phone: 19199668650

Email: gilkey@email.unc.edu

\section{Abstract}

Background: The COVID-19 pandemic has led to unprecedented use of telehealth, including by primary care professionals (PCPs) who serve adolescents.

Objective: To inform future practice and policies, we sought to characterize PCPs' recent experience using adolescent telehealth as well as their support for it after the COVID-19 pandemic is over.

Methods: From February to March 2021, we conducted a web-based survey of 1047 PCPs in the United States. Our national sample included physicians $(747 / 1047,71 \%)$, advanced practice providers $(177 / 1047,17 \%)$, and nurses $(123 / 1047,12 \%)$ who provided primary care to adolescents aged 11-17 years.

Results: Most PCPs reported using telehealth for a low, moderate, or high proportion of their adolescent patients in the three months prior to the survey $(424 / 1047,40 \%, 286 / 1047,27 \%$, and $219 / 1047,21 \%$, respectively); only $11 \%$ (118/1047) reported no use. A majority of respondents agreed that adolescent telehealth increases access to care $(720 / 1047,69 \%)$ and enables them to provide high-quality care $(560 / 1047,53 \%)$. Few believed that adolescent telehealth takes too much time $(142 / 1047,14 \%)$ or encourages health care overuse $(157 / 1047,15 \%)$. Most supported giving families the option of adolescent telehealth for primary care after the pandemic is over $(683 / 1047,65 \%)$ and believed that health insurance plans should continue to reimburse for telehealth visits $(863 / 1047,82 \%)$. Approximately two-thirds $(702 / 1047,67 \%)$ wanted to offer adolescent telehealth visits after the pandemic, with intentions being higher among those with recent telehealth experience $(P<.001)$.

Conclusions: PCPs in our national sample reported widespread use of and predominantly positive attitudes toward adolescent telehealth. Our findings also suggest broad support among PCPs for continuing to offer adolescent telehealth after the COVID-19 pandemic ends.

(J Med Internet Res 2021;23(9):e31240) doi: 10.2196/31240

\section{KEYWORDS}

adolescent health; primary care; telemedicine; health communication; health services; telehealth; adolescent; young adult; teenager; COVID-19; survey; policy; access

\section{Introduction}

The COVID-19 pandemic has rapidly transformed health care delivery in the United States; for the first time, telehealth is playing a central role in the delivery of primary care for adolescents. Research conducted prior to the pandemic suggests that telehealth is a promising mode of delivering discrete types of health care for adolescents, including in the areas of mental 
health, asthma and diabetes management, and gender-affirming care [1-4]. A small body of research also documents the adolescent medicine community's impressive efforts to rapidly scale up telehealth programs in the first months of the pandemic [5-7]. However, to our knowledge, no published national studies have explored the experience of primary care professionals (PCPs) in delivering care to adolescents in the ensuing period, during which telehealth has presumably become a standard offering for many. To address this gap, we surveyed a national sample of PCPs. Our aims were to characterize PCPs' recent adolescent telehealth use and attitudes as well as their support for continuing to offer adolescent telehealth after the COVID-19 pandemic is over.

\section{Methods}

\section{Participants and Procedures}

We conducted a web-based survey of PCPs from February to March 2021. We contracted with a survey research company to administer the survey, which we developed, to a standing national panel. The company maintained the panel using a combination of recruitment methods, including web-based registration, referrals, marketing emails, and digital advertisements. As part of the recruitment process, physicians provided licensure information used to verify their identity. For our survey, eligible panel members were US physicians, advanced practitioners (ie, nurse practitioners and physician assistants), and nurses who provided primary care, including vaccinations, to adolescents aged 11-17 years. In compliance with state policies governing PCPs' survey participation, our sample excluded residents of Vermont.

The survey company emailed invitations and up to two reminders to panel members. A total of 1055 panel members responded by accessing the survey. The response rate was $61 \%$ among physicians and $41 \%$ among advanced practitioners and nurses (American Association for Public Opinion Research response rate 4) [8]. Participants provided informed consent and received up to US $\$ 80$ for their participation, depending on market rates in their area. Based on survey responses, we excluded 8 PCPs who indicated that they saw no adolescent patients in a typical week, resulting in a final sample size of 1047 participants. The University of North Carolina Institutional Review Board approved the study protocol.

\section{Measures}

Our survey began with an introductory statement that defined adolescent telehealth as visits by videoconference or telephone for patients aged 11-17 years. The survey next assessed the extent of PCPs' recent telehealth use with a closed-ended question on the proportion of adolescent patients they saw by telehealth in the 3 months prior to the survey; we recategorized responses as high $(51 \%-75 \%, 76 \%-99 \%$, and $100 \%)$, moderate (26\%-50\%), low (1\%-25\%), or no (0\%) use. Among PCPs with any $(>0 \%)$ use, the survey used 6 closed-ended items to assess telehealth practice. Of these items, 4 used pre-specified lists to assess the type of care provided, perceived advantages, perceived disadvantages, and technological barriers. One item assessed which vaccines PCPs always recommended during telehealth visits for adolescents who were due: seasonal influenza; human papillomavirus (HPV); tetanus, diphtheria, and acellular pertussis (Tdap); and meningococcal ACWY. One item assessed how often PCPs requested confidential time to speak with adolescents during telehealth visits.

Our survey assessed PCPs' perceptions of adolescent telehealth with 7 closed-ended questions that used 5-point response scales, ranging from "strongly disagree" (1) to "strongly agree" (5). Of these items, 4 assessed PCPs' attitudes on whether adolescent telehealth increases access to care, is a way they can provide high-quality care, takes too much time, or encourages health care overuse. A total of 2 items assessed PCPs' support for adolescent telehealth after the COVID-19 pandemic is over in terms of whether families should still have the option to use telehealth for primary care visits and whether health insurance plans should continue to reimburse for visits. One item assessed PCPs' intentions in terms of whether they wanted to offer adolescent telehealth visits once the pandemic is over. For all 7 items, we recategorized responses as disagree ("somewhat" or "strongly"), neither agree or disagree, or agree ("somewhat" or "strongly").

Our survey assessed PCPs' demographic and professional characteristics, including their training, gender, race, number of years in practice, and number of adolescent patients seen in a typical week (Table 1). The survey also assessed characteristics of the clinics in which the PCPs worked. These measures included clinic specialty (family medicine or pediatrics), practice type (solo/group vs other), whether the clinic was part of a health care system or network, the rurality of the area the clinic served, the US census region in which the clinic was located, and the extent to which the clinic experienced financial strain due to the COVID-19 pandemic. 
Table 1. Sample characteristics (N=1047).

\begin{tabular}{|c|c|}
\hline Characteristic & Value, $\mathrm{n}(\%)$ \\
\hline \multicolumn{2}{|c|}{ Primary care professional characteristics } \\
\hline \multicolumn{2}{|l|}{ Training } \\
\hline Physician & $747(71)$ \\
\hline Advanced practice provider ${ }^{\mathrm{a}}$ & $177(17)$ \\
\hline Nurse & $123(12)$ \\
\hline \multicolumn{2}{|l|}{ Gender } \\
\hline Woman & $515(49)$ \\
\hline Man & $492(47)$ \\
\hline Other $^{b}$ & $40(4)$ \\
\hline \multicolumn{2}{|l|}{ Race } \\
\hline White & $717(68)$ \\
\hline Black & $41(4)$ \\
\hline Asian & $170(16)$ \\
\hline Other & $119(11)$ \\
\hline \multicolumn{2}{|l|}{ Years in practice } \\
\hline $0-9$ & $252(24)$ \\
\hline $10-19$ & $395(38)$ \\
\hline 20 or more & $400(38)$ \\
\hline \multicolumn{2}{|c|}{ Adolescent patients seen in a typical week } \\
\hline $1-9$ & $283(27)$ \\
\hline $10-24$ & $431(41)$ \\
\hline 25 or more & $333(32)$ \\
\hline \multicolumn{2}{|c|}{ Proportion of adolescents seen by telehealth in prior 3 months } \\
\hline None $(0 \%)$ & $118(11)$ \\
\hline Low $(1 \%-24 \%)$ & $424(40)$ \\
\hline Moderate $(25 \%-50 \%)$ & $286(27)$ \\
\hline High $(51 \%-100 \%)$ & $219(21)$ \\
\hline \multicolumn{2}{|l|}{ Clinic or practice characteristics } \\
\hline \multicolumn{2}{|l|}{ Specialty } \\
\hline Family medicine & $748(71)$ \\
\hline Pediatrics & $299(29)$ \\
\hline \multicolumn{2}{|l|}{ Practice type } \\
\hline Solo or group & $696(66)$ \\
\hline Other $^{c}$ & $351(34)$ \\
\hline \multicolumn{2}{|l|}{ Part of a health care system } \\
\hline No & 457 (44) \\
\hline Yes & $590(56)$ \\
\hline \multicolumn{2}{|l|}{ Rurality } \\
\hline Urban & $363(35)$ \\
\hline Suburban & $525(50)$ \\
\hline Rural & $159(15)$ \\
\hline
\end{tabular}




\begin{tabular}{ll}
\hline Characteristic & Value, $\mathrm{n}(\%)$ \\
\hline Region & $265(25)$ \\
Northeast & $247(24)$ \\
Midwest & $333(32)$ \\
South & $202(19)$ \\
West & \\
COVID-19-related financial strain & $360(34)$ \\
None or a little & $687(66)$ \\
Moderate to high & \\
\hline
\end{tabular}

${ }^{\mathrm{a}}$ Includes nurse practitioners and physician assistants.

${ }^{\mathrm{b}}$ Includes neither woman nor man, prefer to self-describe, and prefer not to say.

${ }^{\mathrm{c}}$ Includes hospital- and university-affiliated clinics, Federally Qualified Health Centers, and community, public health, and nonprofit clinics.

\section{Statistical Analysis}

We used Pearson chi-square tests to compare the proportions of PCPs who indicated that they always recommended the seasonal influenza vaccine versus each of the other vaccines (HPV, Tdap, meningococcal) during adolescent telehealth visits. We compared the number of advantages and disadvantages that PCPs endorsed for adolescent telehealth using a Wilcoxon signed-rank test. We used bivariate logistic regression to identify correlates of PCPs' intentions to offer adolescent telehealth visits once the COVID-19 pandemic is over, modeling the outcome as yes ("agree") versus no ("neither agree or disagree" and "disagree"). We then simultaneously entered statistically significant correlates into a multivariable model. We conducted analyses using Stata, version 15.1 (StataCorp LLC). Statistical tests were two-tailed with a critical alpha of .05.

\section{Results}

\section{Participant Characteristics}

Our sample of 1047 respondents comprised physicians $(n=747$, $71 \%)$, advanced practitioners $(\mathrm{n}=177,17 \%)$, and nurses $(\mathrm{n}=123$, 12\%; Table 1). Most had 10 or more years of experience in practice $(795 / 1047,76 \%)$ and saw 10 or more adolescent patients in a typical week $(764 / 1047,73 \%)$. PCPs worked in clinics focusing on family medicine $(748 / 1047,71 \%)$ or pediatrics $(299 / 1047,29 \%)$. The clinics were located in all four
US regions; some were in clinical systems or networks (590/1047, 56\%), and some served rural areas $(159 / 1047,15 \%)$. Two-thirds $(687 / 1047,66 \%)$ of PCPs reported that their clinics had experienced moderate to high financial strain due to the COVID-19 pandemic.

\section{Telehealth Practice}

Almost all PCPs reported using telehealth to see adolescent patients in the 3 months prior to the survey (Table 1). Approximately one-fifth of our sample (219/1047, 21\%) indicated high adolescent telehealth use, while others reported more moderate $(286 / 1047,27 \%)$ or low $(424 / 1047,40 \%)$ use. Only $11 \%$ of PCPs $(118 / 1047)$ reported no recent adolescent telehealth use.

The 929 PCPs with recent telehealth visits most often used telehealth for chronic disease management $(n=599,64 \%)$, acute care $(n=571,61 \%)$, mental and behavioral health $(n=561,60 \%)$, or vaccine consultations $(n=406,44 \%)$ (Table 2$)$. Most of these 929 PCPs indicated that they always recommended seasonal influenza vaccination during telehealth visits if adolescents were due ( $\mathrm{n}=798,86 \%)$, but somewhat fewer said the same for HPV, Tdap, and meningococcal vaccines $(\mathrm{n}=715,77 \%, \mathrm{n}=709,76 \%$, and $\mathrm{n}=612,66 \%$, respectively; all $P<.001)$. Approximately one-quarter of these PCPs $(263 / 929,28 \%)$ reported that they "always" or "often" requested time to speak to adolescents confidentially during telehealth visits. 
Table 2. Telehealth practice among primary care professionals with recent adolescent telehealth visits $(\mathrm{n}=929)$.

\begin{tabular}{|c|c|}
\hline & Value, n (\%) \\
\hline \multicolumn{2}{|l|}{ Type of adolescent telehealth visits provided } \\
\hline Chronic disease management & $599(64)$ \\
\hline Acute care & $571(61)$ \\
\hline Mental and behavioral health & $561(60)$ \\
\hline Vaccine consultation & $406(44)$ \\
\hline Other well-child care & $387(42)$ \\
\hline Sexual health and contraceptive counseling & $359(39)$ \\
\hline None of these & $14(2)$ \\
\hline \multicolumn{2}{|l|}{ Vaccines always recommended if due } \\
\hline Seasonal influenza & $798(86)$ \\
\hline Human papillomavirus & $715(77)$ \\
\hline Tetanus, diphtheria, and acellular pertussis & 709 (76) \\
\hline Meningococcal & $612(66)$ \\
\hline None of these & $48(5)$ \\
\hline \multicolumn{2}{|l|}{ Request to speak with adolescent confidentially } \\
\hline Always or often & $263(28)$ \\
\hline Sometimes & $356(38)$ \\
\hline Rarely or never & $310(33)$ \\
\hline \multicolumn{2}{|l|}{ Technology problems ${ }^{a}$} \\
\hline Poor-quality internet connections & $499(85)$ \\
\hline Families' lack of internet-enabled devices & $353(60)$ \\
\hline Lack of training for providers and staff & $195(33)$ \\
\hline Difficulty working with medical interpreters & $128(22)$ \\
\hline None of these & $21(4)$ \\
\hline
\end{tabular}

${ }^{\mathrm{a}}$ Among the subset of participants who reported technology problems as a disadvantage $(\mathrm{n}=584)$.

On average, PCPs reported more advantages than disadvantages of their recent adolescent telehealth use (mean $=3.2$ of 5 advantages, SD 1.3, vs 2.1 of 5 disadvantages, SD $0.9 ; P<.001)$. The most common advantages were preventing COVID-19 exposure $(768 / 929,83 \%)$, putting families at ease $(712 / 929$, $77 \%)$, and reducing families' transportation $(646 / 929,70 \%)$ or time $(639 / 929,69 \%)$ burdens (Figure 1). Only approximately one-quarter of PCPs reported that gaining insight into families' home environments was an advantage (243/929, 26\%). The most common disadvantages were the inability to perform physical examinations $(820 / 929,88 \%)$ and technology problems $(584 / 929,63 \%)$. Only a minority of PCPs indicated that a lack of privacy $(260 / 929,28 \%)$, health insurance problems (191/929, $21 \%$ ), or more missed appointments $(106 / 929,11 \%)$ were disadvantages. Of the 584 PCPs who indicated technology problems as a disadvantage, most reported that poor quality internet connections $(n=499,85 \%)$ and families' lack of internet-enabled devices $(n=353,60 \%)$ were common barriers (Table 2). 
Figure 1. Perceived advantages (A) and disadvantages (B) of adolescent telehealth by primary care providers $(\mathrm{n}=929)$.
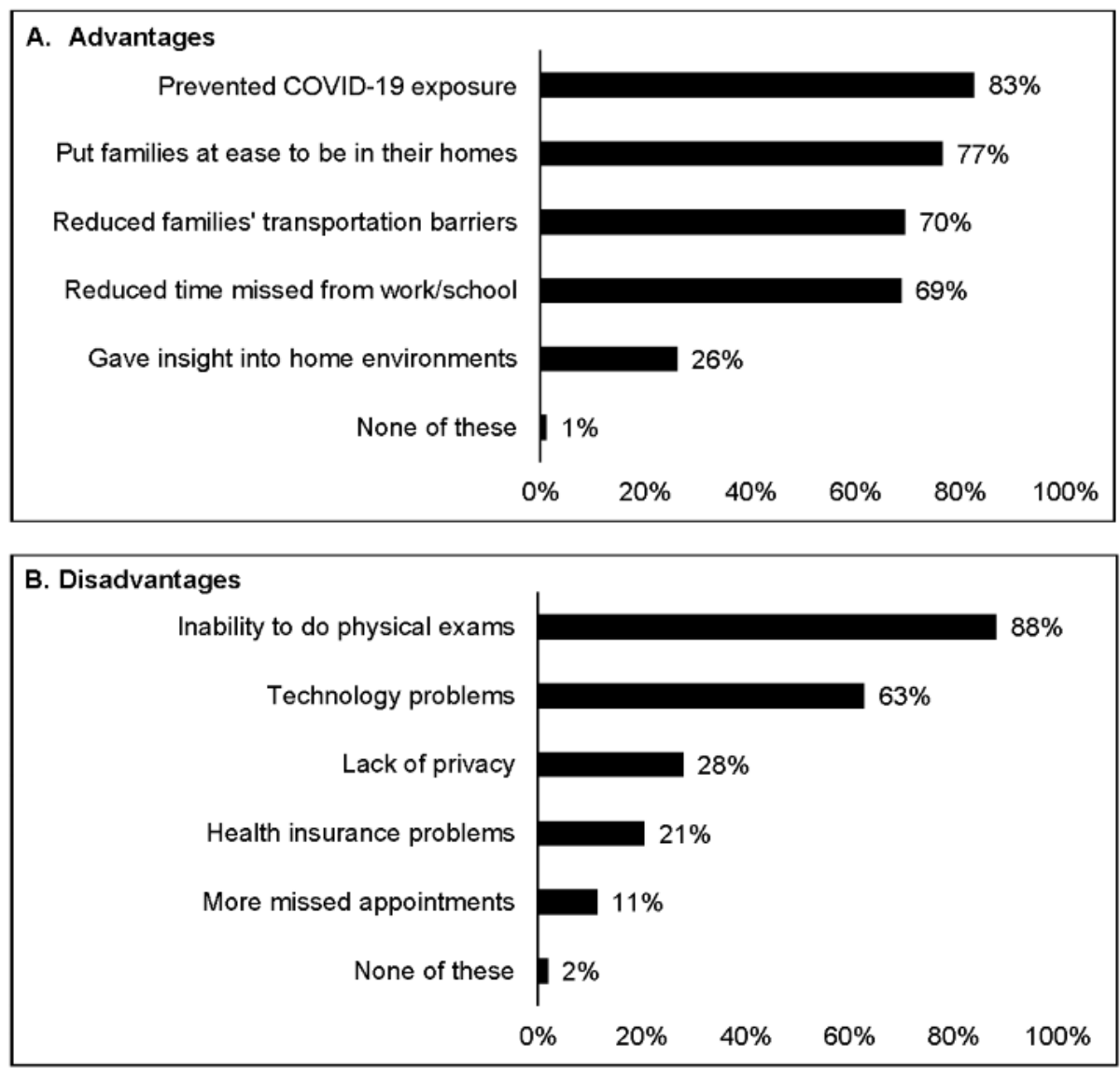

\section{Telehealth Attitudes and Postpandemic Support}

Most PCPs reported positive attitudes toward adolescent telehealth. A majority agreed that telehealth increased access to care for adolescents $(720 / 1047,69 \%)$ and was a way they could provide high-quality care (560/1047, 53\%, Figure 2$)$. Few agreed that telehealth took too much time $(142 / 1047,14 \%)$ or encouraged families to overuse health care for adolescents (157/1047, 15\%).
Most PCPs indicated support for adolescent telehealth after the COVID-19 pandemic is over (Figure 2). Approximately two-thirds $(683 / 1047,65 \%)$ agreed that families should continue to have the option of telehealth for adolescent primary care visits. Approximately four-fifths $(863 / 1047,82 \%)$ agreed that health insurance plans should continue to reimburse for telehealth visits.

Figure 2. Primary care providers' attitudes toward and support for postpandemic adolescent telehealth ( $\mathrm{n}=1047)$.

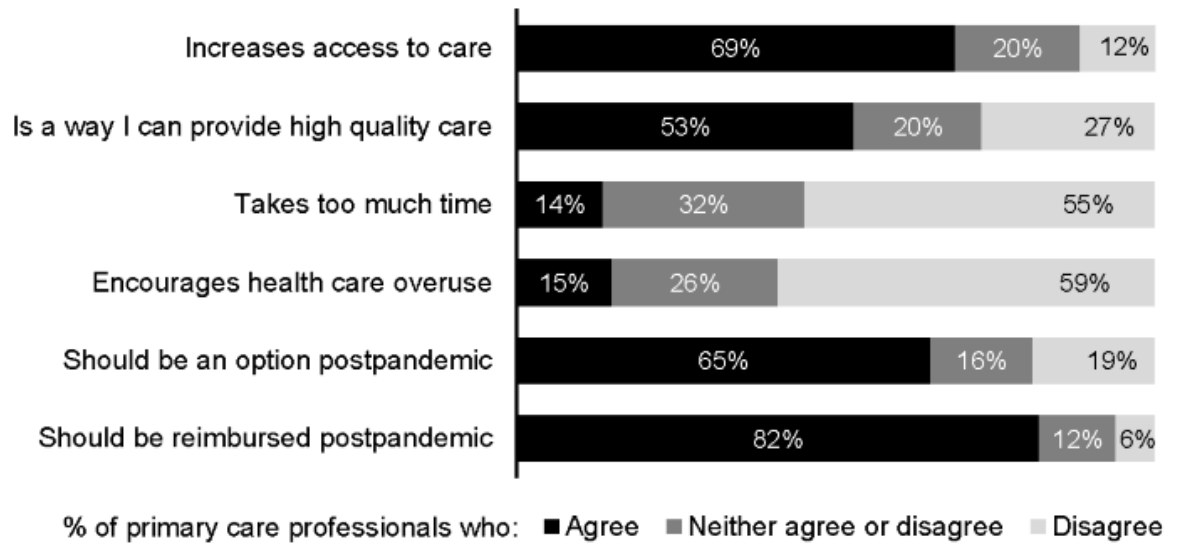




\section{Telehealth Intentions}

Approximately two-thirds of the 1047 PCPs agreed that they want to offer adolescent telehealth visits once the COVID-19 pandemic is over $(\mathrm{n}=702,67 \%)$, while the remainder disagreed $(n=188,18 \%)$ or neither agreed nor disagreed $(n=157,15 \%)$. In multivariable analyses, wanting to offer telehealth was more common among PCPs with high, moderate, and low versus no experience seeing adolescents by telehealth in the 3 months prior to the survey $(175 / 219,80 \%, 211 / 286,74 \%$, and 280/424, $66 \%$, vs $36 / 118,31 \%$, respectively; all $P<.001$ ) (Table 3 ). Wanting to offer telehealth was also more common among PCPs who worked in clinics that served urban versus rural areas $(258 / 363,71 \%$, vs $94 / 159,59 \%, P=.02)$ or that were located in the South or West regions versus the Northeast region $(231 / 333$, $69 \%$, and $143 / 202,71 \%$, vs $161 / 265,61 \% ; P=.003$ and .047 , respectively). Wanting to offer telehealth was less common for PCPs with more years in practice $(262 / 395,66 \%$, for $10-19$ years and $246 / 400,62 \%$, for $\geq 20$ years vs $194 / 252,77 \%$, for $\leq 9$ years; both $P=.02$ ). In bivariate analyses, PCPs' adolescent telehealth intentions correlated with working outside of a solo/group practice or within a health care system, but these associations did not retain statistical significance in the multivariable model. 
Table 3. Correlates of PCPs' intentions to offer adolescent telehealth after the COVID-19 pandemic (N=1047).

\begin{tabular}{|c|c|c|c|c|c|}
\hline & \multirow[t]{2}{*}{$\begin{array}{l}\mathrm{PCPs}^{\mathrm{a}} \text { who want to } \\
\text { offer telehealth, n (\%) }\end{array}$} & \multicolumn{2}{|l|}{ Bivariate analysis } & \multicolumn{2}{|c|}{ Multivariable analysis } \\
\hline & & $\mathrm{OR}^{\mathrm{b}}(95 \% \mathrm{CI})$ & $P$ value & OR $(95 \% \mathrm{CI})$ & $P$ value \\
\hline \multicolumn{6}{|l|}{ PCP characteristics } \\
\hline \multicolumn{6}{|l|}{ Training } \\
\hline Physician (n=747) & $492(66)$ & 1 & $\mathrm{~N} / \mathrm{A}^{\mathrm{c}}$ & $-^{\mathrm{d}}$ & N/A \\
\hline Advanced practice provider $(\mathrm{n}=177)$ & $126(71)$ & $1.28(0.89-1.83)$ & .18 & - & N/A \\
\hline Nurse $(n=123)$ & $84(68)$ & $1.12(0.74-1.68)$ & .60 & - & N/A \\
\hline \multicolumn{6}{|l|}{ Years in practice } \\
\hline $0-9(\mathrm{n}=252)$ & $194(77)$ & 1 & N/A & 1 & N/A \\
\hline $10-19(n=395)$ & $262(66)$ & $0.59(0.41-0.84)$ & .004 & $0.63(0.43-0.92)$ & .02 \\
\hline 20 or more $(n=400)$ & $246(62)$ & $0.48(0.33-0.68)$ & $<.001$ & $0.64(0.44-0.94)$ & .02 \\
\hline \multicolumn{6}{|l|}{ Adolescent patients seen in typical week } \\
\hline $1-9(n=283)$ & $190(67)$ & 1 & N/A & - & N/A \\
\hline $10-24(n=431)$ & $289(67)$ & $1.00(0.72-1.37)$ & .98 & - & N/A \\
\hline 25 or more $(n=333)$ & $223(67)$ & $0.99(0.71-1.39)$ & .96 & - & N/A \\
\hline \multicolumn{6}{|c|}{ Proportion of adolescents seen by telehealth in prior 3 months } \\
\hline None $(0 \%)(\mathrm{n}=118)$ & $36(31)$ & 1 & N/A & 1 & N/A \\
\hline Low $(1 \%-24 \%)(\mathrm{n}=424)$ & $280(66)$ & $4.43(2.85-6.88)$ & $<.001$ & $4.61(2.92-7.27)$ & $<.001$ \\
\hline Moderate $(25 \%-50 \%)(\mathrm{n}=286)$ & $211(74)$ & $6.41(4.00-10.28)$ & $<.001$ & $6.53(4.01-10.64)$ & $<.001$ \\
\hline $\operatorname{High}(51 \%-100 \%)(\mathrm{n}=219)$ & $175(80)$ & $9.06(5.43-15.13)$ & $<.001$ & $8.99(5.31-15.23)$ & $<.001$ \\
\hline \multicolumn{6}{|l|}{ Clinic or practice characteristics } \\
\hline \multicolumn{6}{|l|}{ Specialty } \\
\hline Family medicine $(\mathrm{n}=748)$ & $510(68)$ & 1 & N/A & - & N/A \\
\hline Pediatrics $(n=299)$ & $192(64)$ & $0.84(0.63-1.11)$ & .22 & - & N/A \\
\hline \multicolumn{6}{|l|}{ Practice type } \\
\hline Solo or group $(\mathrm{n}=696)$ & $450(65)$ & 1 & N/A & 1 & N/A \\
\hline Other $(n=351)$ & $252(72)$ & $1.39(1.05-1.84)$ & .02 & $1.16(0.83-1.61)$ & .39 \\
\hline \multicolumn{6}{|l|}{ Part of clinical system } \\
\hline No $(n=457)$ & $280(61)$ & 1 & N/A & 1 & N/A \\
\hline Yes $(n=590)$ & $422(72)$ & $1.59(1.22-2.06)$ & $<.001$ & $1.33(0.98-1.79)$ & .07 \\
\hline \multicolumn{6}{|l|}{ Rurality } \\
\hline Urban $(n=363)$ & $258(71)$ & 1 & N/A & 1 & N/A \\
\hline Suburban $(\mathrm{n}=525)$ & $350(67)$ & $0.81(0.61-1.09)$ & .17 & $0.87(0.63-1.19)$ & .38 \\
\hline Rural (n=159) & $94(59)$ & $0.59(0.40-0.87)$ & .008 & $0.59(0.39-0.90)$ & .02 \\
\hline \multicolumn{6}{|l|}{ Region } \\
\hline Northeast $(n=265)$ & $161(61)$ & 1 & N/A & 1 & N/A \\
\hline Midwest (n=247) & $167(68)$ & $1.35(0.94-1.94)$ & .11 & $1.35(0.92-1.99)$ & .13 \\
\hline South $(n=333)$ & $231(69)$ & $1.46(1.04-2.05)$ & .03 & $1.73(1.20-2.49)$ & .003 \\
\hline West $(n=202)$ & $143(71)$ & $1.57(1.06-2.31)$ & .03 & $1.52(1.01-2.31)$ & .047 \\
\hline \multicolumn{6}{|l|}{ COVID-19-related financial strain } \\
\hline None or a little $(n=360)$ & $241(67)$ & 1 & N/A & - & N/A \\
\hline Moderate or more $(n=687)$ & $461(67)$ & $1.01(0.77-1.32)$ & .96 & - & N/A \\
\hline
\end{tabular}


aCPs: primary care professionals.

${ }^{\mathrm{b}} \mathrm{OR}$ : odds ratio.

${ }^{\mathrm{c}} \mathrm{N} / \mathrm{A}$ : not applicable.

${ }^{\mathrm{d}}$ The variable was not included in the multivariable model because it was not statistically significant at the bivariate level.

\section{Discussion}

\section{Principal Findings}

The findings of our national study suggest that adolescent telehealth has achieved widespread adoption in the year since the COVID-19 pandemic began, with most PCPs in our national study reporting that they used adolescent telehealth and wanted to keep using it. The vast majority (89\%) of PCPs reported using telehealth to see adolescents in the prior 3 months, including for chronic disease management, acute care, and mental health. This level of adoption is far higher than that reported in prepandemic studies, which found that very few pediatricians or family physicians were using telehealth to deliver care $(13 \%$ and $15 \%$, respectively) $[9,10]$. On average, the PCPs in our sample reported that adolescent telehealth offered more advantages than disadvantages, with advantages including increased access to care and reduced time and transportation burdens for families. The most commonly noted disadvantages were the inability to perform physical examinations and technology problems. Despite these limitations, PCPs indicated broad support for adolescent telehealth after the pandemic is over, with approximately two-thirds wanting to offer such visits themselves. This support suggests a pressing need to build on the strengths, address the challenges, and evaluate the quality of adolescent telehealth to ensure it remains a viable option for primary care delivery in the postpandemic era.

Our study provides novel data on two potential challenges for the delivery of adolescent telehealth: vaccine communication and privacy. With regard to vaccine communication, we found that the proportion of PCPs reporting that they "always" recommended vaccines during adolescent telehealth visits was high for seasonal influenza vaccine, perhaps in response to concerns about an influenza-COVID-19 "twindemic." Consistent recommendations were less common for HPV, Tdap, and meningococcal vaccines. In the case of the HPV vaccine, prepandemic studies that used similar measures in the context of traditional primary care documented levels of recommendation consistency that are comparable to or lower than what we observed [11-13]. Although such points of comparison are not available for other adolescent vaccines, our findings suggest that many PCPs are including vaccine counseling in adolescent telehealth visits, and these data can inform interventions to support and further strengthen this communication. For example, PCPs may benefit from electronic health record prompts to remind them to recommend adolescent vaccines during telehealth visits and to counsel families about how to schedule those visits. This care coordination could help to ensure that adolescent telehealth complements rather than competes with the in-person care that is integral to the delivery of vaccinations and other routine preventive health services for adolescents [14].

Consistent with prior studies [5,6], our findings suggest that privacy constitutes an important consideration but may not be a primary barrier to the delivery of adolescent telehealth. Among the PCPs in our sample with recent experience, only approximately one-quarter perceived a lack of privacy as a disadvantage of adolescent telehealth. At the same time, however, only approximately one-quarter routinely offered adolescents confidential time during telehealth visits. Despite being a recommended practice, confidential time is also inconsistently offered during in-person visits $[15,16]$; therefore, this low level of guideline adherence is perhaps unsurprising. Nevertheless, PCPs may have the opportunity to strengthen their telehealth practice by more consistently offering confidential time and by counseling adolescents to take steps that may help protect their privacy, such as using earphones $[5,7,17]$.

Our study can inform future policy making by documenting PCPs' support for offering adolescent telehealth after the COVID-19 pandemic is over. Telehealth adoption during the pandemic has been possible due to expanded payer reimbursement, which prepandemic studies identified as the single largest barrier to bringing pediatric telehealth programs to scale $[9,14,18]$. Importantly, most PCPs in our sample believed that health insurance plans should continue to reimburse for adolescent telehealth after the pandemic is over. Furthermore, many PCPs believed that families should have the option of telehealth for adolescent primary care visits and wanted to offer telehealth visits themselves. Wanting to offer adolescent telehealth was more common among PCPs who had recently used it or who worked in the South or West regions of the United States, but it was less common among those serving rural areas. Future research can extend the present study by assessing factors, such as internet connectivity, that might explain these geographic differences.

\section{Strengths and Limitations}

This study is, to our knowledge, the first national evaluation of PCPs' experience using adolescent telehealth in the context of the COVID-19 pandemic. Study strengths include the use of data from a large, national sample of PCPs, including physicians, advanced practitioners, and nurses. Although evaluating adolescent telehealth from the perspective of those who deliver it is a study strength, our use of self-reported measures also constitutes a limitation. For example, PCPs may have overestimated the consistency with which they recommended vaccines or offered confidential time during adolescent telehealth visits. Another limitation is the modest response rate among advanced practice providers and nurses. Including nonphysician participants was important for enriching our data with diverse perspectives, but additional research with larger sample sizes may be needed to more fully understand how adolescent telehealth practice and attitudes vary across clinical roles. We acknowledge that other perspectives and data sources are important for understanding the impact of adolescent telehealth. Most notably, PCPs in our sample perceived telehealth as expanding access to care and reducing burden on 
their patients, but future research is needed to understand the extent to which adolescents and their families experience telehealth as patient-centered and equitable. Finally, we note that our study examines telehealth broadly as including visits by video and telephone. PCPs' experience of adolescent telehealth may vary between these two delivery modes as well as by health system factors, such as the extent to which telehealth is integrated with electronic health record platforms and patient portals. Future research will be needed to understand the influence of these contextual factors.

\section{Conclusion}

One year into the COVID-19 pandemic, our national study finds that PCPs have widely adopted adolescent telehealth and endorse its continued use. In light of this endorsement, health care system leaders, payers, professional organizations, researchers, and other key stakeholders should redouble their efforts to support PCPs in adolescent telehealth delivery, including by further evaluating and fairly reimbursing such services. In this way, we can ensure that telehealth realizes its potential to increase health care access and to serve adolescents in a way that is effective, patient centered, and equitable.

\section{Data Availability Statement}

Deidentified individual participant data will not be made available.

\section{Acknowledgments}

This project was supported by a Gillings Innovation Laboratory award from the UNC Gillings School of Global Public Health and the Lineberger Comprehensive Cancer Center through a Tier 1 Innovation Award made possible by the University Cancer Research Fund. The authors' time was supported by grants from the National Institutes of Health (T32CA057726 for BKG and K08AI148607 for PT). The funders played no role in the study design; the collection, analysis, and interpretation of data; the writing of the report; or the decision to submit the manuscript for publication.

\section{Conflicts of Interest}

PT receives research funding from the National Institutes of Health and the American Society of Tropical Medicine and Hygiene/Burroughs-Wellcome fund, as well as research support from Abbott Laboratories. NTB has served as a paid advisor to the Centers for Disease Control and Prevention, the World Health Organization, and Merck \& Co, Inc.

\section{References}

1. Myers KM, Valentine JM, Melzer SM. Child and adolescent telepsychiatry: utilization and satisfaction. Telemed J E Health 2008 Mar;14(2):131-137. [doi: 10.1089/tmj.2007.0035] [Medline: 18361702]

2. Sequeira GM, Kidd KM, Coulter RW, Miller E, Fortenberry D, Garofalo R, et al. Transgender youths' perspectives on telehealth for delivery of gender-affirming care. J Adolesc Health 2021 Jun;68(6):1207-1210 [FREE Full text] [doi: 10.1016/j.jadohealth.2020.08.028] [Medline: 32980246]

3. Davies B, Kenia P, Nagakumar P, Gupta A. Paediatric and adolescent asthma: a narrative review of telemedicine and emerging technologies for the post-COVID-19 era. Clin Exp Allergy 2021 Mar 10;51(3):393-401 [FREE Full text] [doi: 10.1111/cea.13836] [Medline: $\underline{33527625]}$

4. Wood CL, Clements SA, McFann K, Slover R, Thomas JF, Wadwa RP. Use of telemedicine to improve adherence to American Diabetes Association Standards in pediatric type 1 diabetes. Diabetes Technol Ther 2016 Jan;18(1):7-14. [doi: 10.1089/dia.2015.0123] [Medline: 26295939]

5. Wood SM, White K, Peebles R, Pickel J, Alausa M, Mehringer J, et al. Outcomes of a rapid adolescent telehealth scale-up during the COVID-19 pandemic. J Adolesc Health 2020 Aug;67(2):172-178 [FREE Full text] [doi:

10.1016/j.jadohealth.2020.05.025] [Medline: 32611509]

6. Barney A, Buckelew S, Mesheriakova V, Raymond-Flesch M. The COVID-19 pandemic and rapid implementation of adolescent and young adult telemedicine: challenges and opportunities for innovation. J Adolesc Health 2020 Aug;67(2):164-171 [FREE Full text] [doi: 10.1016/j.jadohealth.2020.05.006] [Medline: 32410810]

7. Carlson JL, Goldstein R. Using the electronic health record to conduct adolescent telehealth visits in the time of COVID-19. J Adolesc Health 2020 Aug;67(2):157-158 [FREE Full text] [doi: 10.1016/j.jadohealth.2020.05.022] [Medline: 32517972]

8. Standard Definitions: Final Dispositions of Case Codes and Outcome Rates for Surveys. The American Association for Public Opinion Research. 2016. URL: https://www.aapor.org/AAPOR Main/media/publications/ Standard-Definitions20169theditionfinal.pdf [accessed 2021-08-30]

9. Sisk B, Alexander J, Bodnar C, Curfman A, Garber K, McSwain SD, et al. Pediatrician attitudes toward and experiences with telehealth use: Results from a national survey. Acad Pediatr 2020 Jul;20(5):628-635 [FREE Full text] [doi: 10.1016/j.acap.2020.05.004] [Medline: 32437881]

10. Klink K, Coffman M, Moore M, Jetty A, Petterson S, Bazemore A. Family Physicians and Telehealth: Findings from a National Survey. Robert Graham Center. 2015 Oct 30. URL: https://www.graham-center.org/content/dam/rgc/documents/ publications-reports/reports/RGC\%202015\%20Telehealth\%20Report.pdf [accessed 2021-08-30] 
11. Finney Rutten LJ, St Sauver JL, Beebe TJ, Wilson PM, Jacobson DJ, Fan C, et al. Association of both consistency and strength of self-reported clinician recommendation for HPV vaccination and HPV vaccine uptake among 11- to 12-year-old children. Vaccine 2017 Oct 27;35(45):6122-6128 [FREE Full text] [doi: 10.1016/j.vaccine.2017.09.056] [Medline: 28958810]

12. Vadaparampil ST, Malo TL, Sutton SK, Ali KN, Kahn JA, Casler A, et al. Missing the target for routine human papillomavirus vaccination: consistent and strong physician recommendations are lacking for 11- to 12-year-old males. Cancer Epidemiol Biomarkers Prev 2016 Aug 02;25(10):1435-1446. [doi: 10.1158/1055-9965.epi-15-1294]

13. Gilkey MB, Malo TL, Shah PD, Hall ME, Brewer NT. Quality of physician communication about human papillomavirus vaccine: findings from a national survey. Cancer Epidemiol Biomarkers Prev 2015 Nov;24(11):1673-1679 [FREE Full text] [doi: $\underline{10.1158 / 1055-9965 . E P I-15-0326}$ ] [Medline: 26494764]

14. Fiks AG, Jenssen BP, Ray KN. A defining moment for pediatric primary care telehealth. JAMA Pediatr 2021 Jan 01;175(1):9-10. [doi: 10.1001/jamapediatrics.2020.1881] [Medline: $\underline{32658256]}$

15. Sieving RE, Mehus C, Catallozzi M, Grilo S, Steiner RJ, Brar P, et al. Understanding primary care providers' perceptions and practices in implementing confidential adolescent sexual and reproductive health services. J Adolesc Health 2020 Oct;67(4):569-575. [doi: 10.1016/j.jadohealth.2020.03.035] [Medline: 32389456]

16. Grilo SA, Catallozzi M, Santelli JS, Yan H, Song X, Heitel J, et al. Confidentiality discussions and private time with a health-care provider for youth, United States, 2016. J Adolesc Health 2019 Mar;64(3):311-318. [doi: 10.1016/j.jadohealth.2018.10.301] [Medline: 30638752]

17. McSwain SD, Bernard J, Burke BL, Cole SL, Dharmar M, Hall-Barrow J, et al. American Telemedicine Association operating procedures for pediatric telehealth. Telemed J E Health 2017 Sep;23(9):699-706. [doi: 10.1089/tmj.2017.0176] [Medline: 28829680]

18. Olson CA, McSwain SD, Curfman AL, Chuo J. The current pediatric telehealth landscape. Pediatrics 2018 Mar;141(3):e20172334 [FREE Full text] [doi: 10.1542/peds.2017-2334] [Medline: 29487164]
Abbreviations
HPV: human papillomavirus
PCP: primary care professional
Tdap: tetanus, diphtheria, and acellular pertussis

Edited by C Basch; submitted 14.06.21; peer-reviewed by A Barney, M Salimi; comments to author 01.07.21; revised version received
10.07.21; accepted 14.07.21; published 10.09.21
Please cite as:
Gilkey MB, Kong WY, Huang Q, Grabert BK, Thompson P, Brewer NT
Using Telehealth to Deliver Primary Care to Adolescents During and After the COVID-19 Pandemic: National Survey Study of US
Primary Care Professionals
J Med Internet Res $2021 ; 23(9):$ e31240
URL: $\underline{\text { htps://www.jmir.org/2021/9/e31240 }}$
doi: $\underline{10.2196 / 31240}$
PMID: $\underline{34406974}$

CMelissa B Gilkey, Wei Yi Kong, Qian Huang, Brigid K Grabert, Peyton Thompson, Noel T Brewer. Originally published in the Journal of Medical Internet Research (https://www.jmir.org), 10.09.2021. This is an open-access article distributed under the terms of the Creative Commons Attribution License (https://creativecommons.org/licenses/by/4.0/), which permits unrestricted use, distribution, and reproduction in any medium, provided the original work, first published in the Journal of Medical Internet Research, is properly cited. The complete bibliographic information, a link to the original publication on https://www.jmir.org/, as well as this copyright and license information must be included. 